\title{
Sensory noise explains auditory frequency discrimination learning induced by training with identical stimuli
}

\author{
Christophe Micheyl, Josh H. MCDermott, AND ANDRew J. OXenham \\ University of Minnesota, Minneapolis, Minnesota
}

\begin{abstract}
Thresholds in various visual and auditory perception tasks have been found to improve markedly with practice at intermediate levels of task difficulty. Recently, however, there have been reports that training with identical stimuli, which, by definition, were impossible to discriminate correctly beyond chance, could induce as much discrimination learning as could training with different stimuli. These surprising findings have been interpreted as evidence that discrimination learning can occur in the absence of perceived differences between stimuli and need not involve the fine-tuning of a discrimination mechanism. Here, we show that these counterintuitive findings of discrimination learning without discrimination can be understood simply by considering the effect of internal noise on sensory representations. Because of such noise, physically identical stimuli are unlikely to be perceived as being strictly identical. We show that, given empirically derived levels of sensory noise, perceived differences evoked by identical stimuli are actually not much smaller than those induced by the physical differences typically used in discrimination-learning experiments. We suggest that findings of discrimination learning with identical stimuli can be explained without implicating any fundamentally new learning mechanism.
\end{abstract}

Improvements in performance with practice have been demonstrated to occur in many visual (e.g., Dosher \& Lu, 1998, 1999, 2005, 2006; Fahle \& Morgan, 1996; Karni \& Sagi, 1991, 1993; Lu \& Dosher, 2004; Poggio, Fahle, \& Edelman, 1992; Schoups \& Orban, 1996; Schoups, Vogels, Qian, \& Orban, 2001) and auditory (e.g., Amitay, Hawkey, \& Moore, 2005; Delhommeau, Micheyl, \& Jouvent, 2005; Demany, 1985; Demany \& Semal, 2002; Hawkey, Amitay, \& Moore, 2004; Irvine, Martin, Klimkeit, \& Smith, 2000; Micheyl, Delhommeau, Perrot, \& Oxenham, 2006; Mossbridge, Fitzgerald, O'Connor, \& Wright, 2006; Roth, Refael-Taub, Sharvit, \& Kishon-Rabin, 2006; Wright, 2001; Wright, Buonomano, Mahncke, \& Merzenich, 1997; Wright \& Sabin, 2007) perception tasks. A widespread view is that optimal perceptual learning is achieved through training at a level that is neither too easy nor too difficult (Ahissar \& Hochstein, 1997). If the task is too easy, participants may not devote enough attention to it; if it is too difficult, they may never grasp it. Accordingly, in most studies of perceptual learning, participants are trained at intermediate levels of difficulty - typically, producing performance of around $75 \%$ correct.

This common assumption was recently challenged by demonstrations that training on an auditory frequency discrimination task with identical tones (by definition, impossible to discriminate correctly) nonetheless resulted in improved discrimination thresholds (Amitay, Irwin, \& Moore, 2006; Roth et al., 2006). Remarkably, the improvement was comparable to that achieved by training with tones that physically differed in frequency. These surprising findings have been interpreted as evidence that discrimination learning can occur without perceived differences between the stimuli (Roth et al., 2006) and, thus, "need not involve fine-tuning a stimulus comparison mechanism" (Amitay et al., 2006, p. 1147). Instead, it was proposed that training might merely improve the ability to "attend to a task-specific stimulus dimension" or to "access a low-level representation and make it available for further processing" (Amitay et al., 2006, p. 1147).

Here, we suggest that although these findings may seem counterintuitive, they can be reconciled simply with more traditional accounts of learning by considering the effects of random variability in neural responses to sensory stimuli - a form of internal noise (Green \& Swets, 1966). Because of this random variability, sensory responses to different presentations of the same stimuli are rarely identical but, rather, vary from one presentation to the next. Thus, from the point of view of the observer, there may be no qualitative distinction between identical and different stimuli, particularly when stimulus differences are close to threshold, as they usually are in psychophysical experiments that seek to measure thresholds. We hypothesized 
that the differences produced by internal noise might be large enough to fine-tune the relevant sensory comparison mechanism and, thus, produce learning. To examine how large such differences might be, we computed the distributions of differences that would be experienced by an observer in Amitay et al.'s (2006) experiment, assuming Gaussian-distributed internal noise with equal variance. The noise level can be straightforwardly derived from discrimination thresholds under the assumptions of signal detection theory.

Amitay et al.'s (2006) experiment involved an oddball paradigm: Listeners were presented on each trial with three tones, the frequency of one of which could differ from that of the other two; listeners were instructed to "pick the odd one out." Depending on the condition, training was performed with the frequency difference either fixed at $0 \mathrm{~Hz}$ (identical stimuli, producing a chance performance of $33 \%$ correct) or varied adaptively to track the $50 \%$ correct, $75 \%$ correct, or $95 \%$ correct threshold. Learning was assessed with a probe condition that adaptively tracked $79 \%$ correct, via the threshold reduction between pre- and posttraining sessions.

Following classical signal detection theory (Green \& Swets, 1966; Macmillan \& Creelman, 2005; Wickens, 2001), we assumed that the sensory observation (perceived frequency), $X_{i}$, evoked by each tone was independently contaminated by additive Gaussian internal noise. We drew samples from these Gaussian random variables to simulate trials of the experiment. The noise level $\sigma$ was calculated from the mean pretraining threshold across all groups (approximately $15 \mathrm{~Hz}$ ), using the basic equation of signal detection theory: $d^{\prime}=\Delta_{\text {thresh }} / \sigma$, where $\Delta_{\text {thresh }}$ is the measured threshold and $d^{\prime}$ is the observer's sensitivity, as determined by the psychophysical paradigm and targeted percent correct ( $79 \%$ correct in the three-interval paradigm corresponds to a $d^{\prime}$ of 1.63). To simulate the conditions with physically different stimuli, the expected value of one of the $X_{i}$ s (chosen at random on each trial) exceeded the other two by $d^{\prime} \sigma$, where $d^{\prime}$ was set to achieve $50 \%, 75 \%, 79 \%$, or $95 \%$ correct, as in Amitay et al.'s (2006) experiment. For the identical-stimuli condition, $d^{\prime}$ was set to zero.

Figure 1 plots the resulting distributions of difference magnitudes between sensory observations $\left(Y=\left\{\left|X_{2}-X_{1}\right|\right.\right.$, $\left.\left.\left|X_{3}-X_{2}\right|,\left|X_{3}-X_{1}\right|\right\}\right)$ in the different conditions. One can see that the distribution for identical stimuli $(0 \mathrm{~Hz})$ overlaps substantially with those for the physically different stimuli that produce above-chance performance. "Identical" stimuli can thus often produce substantial apparent frequency differences.

In order to illustrate how large the perceived differences evoked by identical stimuli could be, we converted the dimensionless internal $z$ scale (which was used originally to compute the probability distributions shown in Figure 1) into physical hertz units. The conversion was made using the equation $\sigma=\Delta_{\text {thresh }} / d^{\prime}$, where $\Delta_{\text {thresh }}$ was taken to be the mean pretraining threshold measured by Amitay et al. (2006) $(14.64 \mathrm{~Hz})$, and $d^{\prime}$ was set to 1.63 (corresponding to $79 \%$ correct in the three-interval paradigm used by these authors). The resulting hertz values are shown along

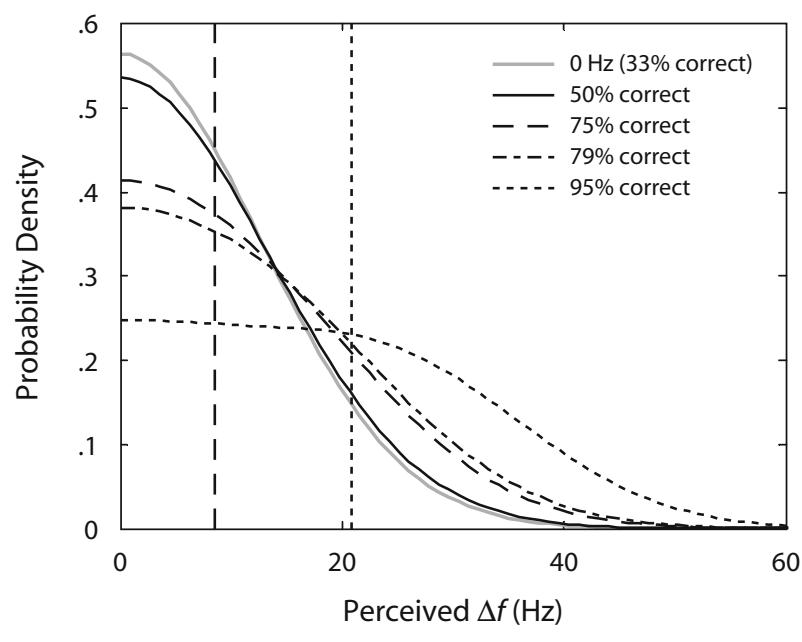

Figure 1. Hypothetical distributions of perceived frequency differences evoked by physical frequency differences $(\Delta f)$ corresponding to different percentages of correct responses in Amitay, Irwin, and Moore's (2006) frequency discrimination experiment. The percent-correct levels are the same as those used by Amitay et al. (2006); they range from chance (33\% correct) for the identical-frequency tones $(\Delta f=0 \mathrm{~Hz})$ to well above chance $(95 \%$ correct). The distributions of perceived differences were derived under the assumption of classical psychophysical signal detection theory, according to which the sensory observations evoked by each stimulus are contaminated independently by constant-variance Gaussian noise with zero mean. The standard deviation of the noise was estimated on the basis of the pretraining thresholds reported by Amitay et al. (2006; see the text for details). The vertical long- and short-dashed lines denote the 50th and 90th percentiles, respectively, of the identical-stimuli $(\Delta f=$ $0 \mathrm{~Hz}$ ) distribution.

the $x$-axis in Figure 1. The vertical long- and short-dashed lines mark the points corresponding to the 50th and 90th percentiles of the identical-stimuli $(0-\mathrm{Hz})$ distribution. As can be seen, according to these simple calculations, identical stimuli elicited perceived differences corresponding to physical differences larger than about $8.5 \mathrm{~Hz}$ (longdashed line) on $50 \%$ of the trials; on approximately $10 \%$ of the trials, the differences were larger than $20 \mathrm{~Hz}$. It is not inconceivable that such perceived differences were sufficient to induce significant learning.

Although relating internal noise or perceiveddifference distributions to physical stimulus values requires deriving a specific internal noise level from thresholds, it is worth noting that the overlap between the distributions for different percentages of correct responses is actually independent of the noise level. Thus, even if it is assumed that the internal noise that limits discrimination performance decreases during learning as thresholds improve, as long as all of the distributions of internal noise are scaled by the same factor, the degree of overlap between them remains constant. Whether frequency discrimination learning results from decreasing internal noise in the sensory representation of tones or can be explained by other factors is not currently known. Examples of perceptual learning not accompanied by decreases in internal noise have been identified in vision (Gold, Bennett, \& Sekuler, 1999). 
These observations demonstrate that variability in responses to identical stimuli can be on a par with response differences produced by physical stimulus variation. This provides a simple explanation for the finding that participants can learn to discriminate stimuli more accurately even when they are trained with stimuli that do not vary along the relevant physical dimension. It also provides a way of reconciling these findings with others in the literature (e.g., Dosher \& Lu, 1998, 1999, 2005, 2006; Lu \& Dosher, 2004), which suggest that practicerelated performance improvements in perceptual detection or discrimination tasks are due, at least in part, to the progressive fine-tuning of those sensory detection and/or discrimination mechanisms that are repeatedly engaged during training.

\section{AUTHOR NOTE}

This work was supported by NIH Grant R01 DC 05216 . The authors are grateful to Sygal Amitay, David Moore, and three anonymous reviewers for comments on an earlier version of the manuscript. Address correspondence to C. Micheyl, Auditory Perception and Cognition Laboratory, Department of Psychology, University of Minnesota, N640b Elliott Hall, 75 East River Road, Minneapolis, MN 55455-0344 (e-mail: cmicheyl@umn.edu).

\section{REFERENCES}

Ahissar, M., \& Hochstein, S. (1997). Task difficulty and the specificity of perceptual learning. Nature, 387, 401-406.

Amitay, S., Hawkey, D. J., \& Moore, D. R. (2005). Auditory frequency discrimination learning is affected by stimulus variability. Perception \& Psychophysics, 67, 691-698.

Amitay, S., Irwin, A., \& Moore, D. R. (2006). Discrimination learning induced by training with identical stimuli. Nature Neuroscience, 9, 1446-1448

Delhommeau, K., Micheyl, C., \& Jouvent, R. (2005). Generalization of frequency discrimination learning across frequencies and ears: Implications for underlying neural mechanisms in humans. Journal of the Association for Research in Otolaryngology, 6, 171-179.

Demany, L. (1985). Perceptual learning in frequency discrimination. Journal of the Acoustical Society of America, 78, 1118-1120.

Demany, L., \& Semal, C. (2002). Learning to perceive pitch differences. Journal of the Acoustical Society of America, 111, 1377-1388.

Dosher, B. A., \& Lu, Z. L. (1998). Perceptual learning reflects external noise filtering and internal noise reduction through channel reweighting. Proceedings of the National Academy of Sciences, 95, 13988-13993.

Dosher, B. A., \& Lu, Z. L. (1999). Mechanisms of perceptual learning. Vision Research, 39, 3197-3221.

Dosher, B. A., \& Lu, Z. L. (2005). Perceptual learning in clear displays optimizes perceptual expertise: Learning the limiting process. Proceedings of the National Academy of Sciences, 102, 5286-5290.

Dosher, B. A., \& LU, Z. L. (2006). Level and mechanisms of perceptual learning: Learning first-order luminance and second-order texture objects. Vision Research, 46, 1996-2007.

Fahle, M., \& Morgan, M. (1996). No transfer of perceptual learning between similar stimuli in the same retinal position. Current Biology, 6, 292-297.

Gold, J., Bennett, P. J., \& Sekuler, A. B. (1999). Signal but not noise changes with perceptual learning. Nature, 402, 176-178.

Green, D. M., \& Swets, J. A. (1966). Signal detection theory and psychophysics. New York: Krieger.

HaWkey, D. J., Amitay, S., \& Moore, D. R. (2004). Early and rapid perceptual learning. Nature Neuroscience, 7, 1055-1056.

Irvine, D. R., Martin, R. L., Klimkeit, E., \& SMith, R. (2000). Specificity of perceptual learning in a frequency discrimination task. Journal of the Acoustical Society of America, 108, 2964-2968.

KARNI, A., \& SAGI, D. (1991). Where practice makes perfect in texture discrimination: Evidence for primary visual cortex plasticity. Proceedings of the National Academy of Sciences, 88, 4966-4970.

KARNI, A., \& SAGI, D. (1993). The time course of learning a visual skill. Nature, 365, 250-252.

Lu, Z. L., \& Dosher, B. A. (2004). Perceptual learning retunes the perceptual template in foveal orientation identification. Journal of Vision, 4, 44-56.

Macmillan, N. A., \& Creelman, C. D. (2005). Detection theory: A user's guide (2nd ed.). Mahwah, NJ: Erlbaum.

Micheyl, C., Delhommeau, K., Perrot, X., \& Oxenham, A. J. (2006) Influence of musical and psychoacoustical training on pitch discrimination. Hearing Research, 219, 36-47.

Mossbridge, J. A., Fitzgerald, M. B., O'Connor, E. S., \& Wright, B. A. (2006). Perceptual-learning evidence for separate processing of asynchrony and order tasks. Journal of Neuroscience, 26, $12708-12716$

Poggio, T., Fahle, M., \& Edelman, S. (1992). Fast perceptual learning in visual hyperacuity. Science, 256, 1018-1021.

Roth, D. A., Refael-Taub, R., Sharvit, R., \& Kishon-Rabin, L. (2006). Can learning a frequency discrimination task occur without discrimination? Journal of Basic Clinical Physiology \& Pharmacology, 17, 159-171.

Schoups, A. A., \& Orban, G. A. (1996). Interocular transfer in perceptual learning of a pop-out discrimination task. Proceedings of the National Academy of Sciences, 93, 7358-7362.

Schoups, A. [A.], Vogels, R., Qian, N., \& Orban, G. (2001). Practising orientation identification improves orientation coding in V1 neurons. Nature, 412, 549-553.

WicKens, T. (2001). Elementary signal detection theory. Oxford: Oxford University Press.

Wright, B. A. (2001). Why and how we study human learning on basic auditory tasks. Audiology \& Neurotology, 6, 207-210.

Wright, B. A., Buonomano, D. V., Mahncke, H. W., \& Merzenich, M. M. (1997). Learning and generalization of auditory temporalinterval discrimination in humans. Journal of Neuroscience, $\mathbf{1 7}$, 3956-3963.

Wright, B. A., \& Sabin, A. T. (2007). Perceptual learning: How much daily training is enough? Experimental Brain Research, 180, 727-736.

(Manuscript received June 9, 2008; accepted for publication July 24, 2008.) 